Haya: The Saudi Journal of Life Sciences

Abbreviated Key Title: Haya Saudi J Life Sci ISSN 2415-623X (Print) |ISSN 2415-6221 (Online) Scholars Middle East Publishers, Dubai, United Arab Emirates Journal homepage: https://saudijournals.com/sjls

Original Research Article

\title{
Photophysical Studies of 4-Dicyanomethylene 2, 6-Dimethyl-4H-Pyran (DDP) Dye with Bovine Serum Albumin (BSA) in Aqueous Solution
}

Thamarai Selvan, Ramachandran, Gayathri Somasundaram, Vasanthi Rajaraman, Vanjinathan Mahalingam, Rajendran Kumaran

Department of Chemistry, Dwaraka Doss Goverdhan Doss Vaishnav College (Autonomous) (Affiliated to University of Madras) 833, Gokul Bagh, E.V.R.Periyar Road, Arumbakkam, Chennai 600 106, Tamil Nadu, India

DOI: $10.36348 /$ sjls.2020.v05i04.001

| Received: 13.04.2020 | Accepted: 20.04.2020 | Published: 22.04.2020

*Corresponding author: Rajendran Kumaran

\section{Abstract}

Photophysical techniques were employed as a tool to determine the binding interaction of an Intramolecular Charge Transfer (ICT) based dye 4- Dicyanomethylene-2,6-dimethyl-4H-pyran (DDP) with Bovine Serum Albumin (BSA) in water. Addition of BSA to DDP dye results no significant change in the ICT absorption maximum but a well-marked enhancement in the fluorescence quantum yield of the dye. The enhancement on the addition of BSA shows that the excited state characteristics of dye are influenced by the protein that comprises several hydrophilic and hydrophobic amino acid moieties. Fluorescence lifetime decay characteristics of DDP dye with BSA exhibits a tri-exponential decay with a large variation in the fluorescence lifetime and relative amplitude distribution. The dye is situated predominantly in the hydrophobic interior rather in the aqueous phase is established from time resolved fluorescence lifetime studies. The coexistence of three different fluorescence lifetime components of dye in the presence of BSA signifies the existence of dye in a heterogeneous micro environment with varying proportion. The existence of multi environment is authenticated by electrochemical impedance spectral studies (EIS). BSA markedly influences the redox potential properties of dye which reveals that the presence of charged species (zwitter ionic structure of amino acids), polar and non-polar moieties governs the electrochemical nature of DDP dye.

Keywords: DDP; BSA; amino acids; fluorescence emission; fluorescence lifetime; fluorescence enhancement: binding plot; electrochemical impedance spectra.

Copyright @ 2020: This is an open-access article distributed under the terms of the Creative Commons Attribution license which permits unrestricted use, distribution, and reproduction in any medium for non-commercial use (NonCommercial, or CC-BY-NC) provided the original author and source are credited.

\section{INTRODUCTION}

Bovine Serum Albumin is a large globular protein that has a better solubility in water. The molecular weight of BSA is around $66.4 \mathrm{kD}$ consisting of 582 amino acids and it contains a single polypeptide chain folded into tertiary globular conformation forming three domains [1]. BSA and HSA are homologous in nature and the amino acid sequence of HSA shows structural similarity with that of BSA [2, 3]. The structure of HSA is well resolved and the modified version adapted from Protein Data Bank (HSA: 1BKE.pdb. PDB:http://www.rscb.org.pdb [4]) is shown in figure 1 .

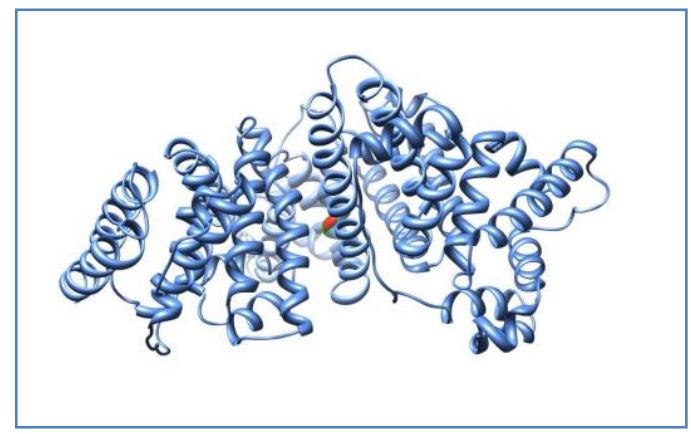

Fig-1: Structure of HSA (adapted and modified from PDB: HSA: 1BKE)[4] 
Serum albumin, the most widely studied globular protein is present in blood plasma and in circulatory system $[5,6]$. BSA consists of a micro heterogeneous population of proteins, constituting 85$95 \%$ monomer, 5-10 \% dimers, and polymers constituting a smaller proportion [7, 8] such that it possesses both hydrophilic and hydrophobic properties. The native structure of BSA contains $66 \% \alpha$-helix and $33 \%$ of $\beta$-structure and the tertiary structure is characterized by three domains I, II and III. The existence of these domains was determined by X-ray crystallography studies on HAS [5, 6]. BSA involves in the transportation of fatty acids, amino acids, steroids and metal ions and is responsible for the maintenance of blood $\mathrm{pH}[5,9]$. Further, BSA binds with ligands and contributes to $80 \%$ of the osmotic blood pressure [5]. An important application of BSA in the biomedical field involving BSA is that it forms stable complexes with drugs and extrinsic probes, [11-33] such that a large variation in the photophysical properties are monitored which are widely useful for chemists and biologists.

BSA has a larger proportion of hydrophobic interior and less non-polar surface at the exterior; thereby the protein-protein interactions in aqueous solutions are significantly important. The nature of interaction in protein depends upon the number of charged groups and non-polar groups exposed in the aqueous medium and buried in the hydrophobic interior $[4,13]$. In BSA, the binding affinity offered by site (I) is mainly through hydrophobic interactions, whereas site (II) involves a combination of hydrophobic interactions, hydrogen bonding and electrostatic interactions $[12,13]$.The hydrogen-bonding interactions involving BSA in aqueous solution and the presence of the hydrophobic bond in proteins [14], peptides have a contrasting effect on the structure of water. Further the interaction of BSA with several extrinsic probes have been explored in depth exhibiting Intramolecular Charge Transfer (ICT), Photoinduced Electron Transfer (PET), Twisted Intramolecular Charge Transfer (TICT) and Excited State Intramolecular Proton Transfer (ESIPT) based dyes which portrays BSA as an ideal host molecule. Further, fluorescence techniques provides several advantages over other analytical techniques in the determining the nature of binding. Photophysical and photochemical properties of probe or drug are monitored by fluorescence spectroscopic techniques, which is so far the most efficient and much reliable tool for the chemists and biologists in particular [2].

Fluorescence spectroscopy is the technique involved in establishing the location of a fluorescent probe in micro heterogeneous environment like protein and peptide aggregates. The photophysical properties of the fluorescent probe are attributed to the micro and macro environment of the medium. Since most of the reactions occur in the time scale ranging from few picoseconds to microseconds in the solution phase, fluorescence spectroscopic techniques are widely used in establishing the properties of the fluorescent probes. Further, a low concentration (in $\mu \mathrm{M}$ ) of the probe is only required to establish the spectral properties.

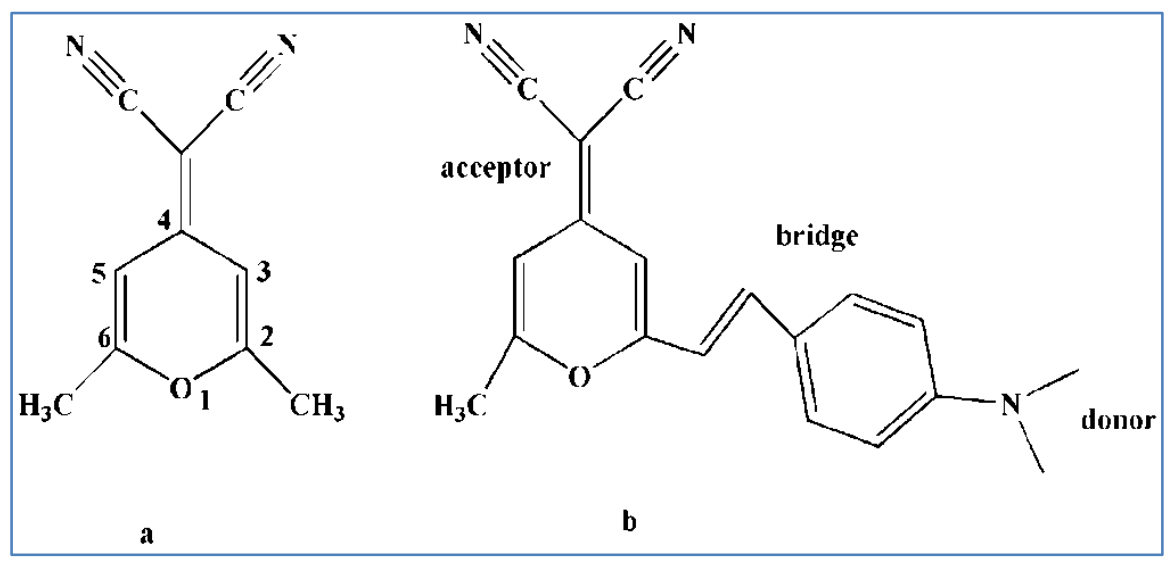

Fig-2: Structural representation of DDP (2a) and DCM (2b) dyes

DDP belongs to the family of DCM type dyes which belongs to the class of red luminescence compound [34-38]. DDP dye has a strong acceptor group dicyanomethylene $-\mathrm{C}(\mathrm{CN})_{2}$ in $4^{\text {th }}$ position and donor moieties in $2^{\text {nd }}$ and $6^{\text {th }}$ positions as shown in figure 2. The photophysical studies were confined predominantly to DCM dye owing to the Twisted Intramolecular Charge Transfer (TICT) behavior [3446] and DDP dye does not exhibit TICT phenomenon. So far DDP dye has been employed as a fluorescent probe to explore the photophysical and electrochemical properties in the presence of hydrogen-bonding solutes such that the interaction at the bulk and the interface regions are well understood.

In our present study, we focus on the variation in the photophysical properties of an ICT based dye, 4Dicyanomethylene-2, 6-dimethyl-4H-pyran (DDP) in the presence of BSA in water. The reports on DDP dye with hydrogen-bonding molecules [44] in aqueous solution 
which portray that the photophysical properties of DDP dye are largely governed by the hydrogen-bonding properties as well as the hydrophobic nature of amide derivatives [42] and $t$ urea derivatives [45]. Further, the studies pertaining exclusively to the fluorescence emission and lifetime of DDP dye in the presence of water soluble non fluorescent solutes are very limited in the literature to the best of our knowledge [42-45].

\section{EXPERIMENTAL METHODS Materials}

BSA fraction $\mathrm{V}$ powder $\mathrm{pH}-7$, was purchased from SRL Chemicals India Ltd., and was refrigerated at $4^{\circ} \mathrm{C}$ throughout our studies. DDP dye was prepared by the procedure reported in the literature [34].

\section{Steady-state absorption and fluorescence measurements}

The absorption spectra were recorded using an Agilent 8453 UV-Visible diode array spectrophotometer. The lamp source used for the ultraviolet-visible range was deuterium and tungsten lamps. Emission spectra and 3D spectral measurements were recorded in Fluoromax-4P spectrofluorimeter (Horiba JobinYvon) using fluorescence software provided by the manufacturer. 3D Contour plots were obtained by the simultaneously scanning the excitation and emission monochromator. Steady state fluorescence anisotropy measurements were carried out in PerkinElmer MPF-44B fluorescence spectrophotometer interfaced with PC through Rishcom-100 multimeter. Sheet polarizer was used in anisotropy measurements and the experimental setup was followed as reported [46].

\section{Time-resolved fluorescence measurements-Time correlated single-photon counting (TCSPC) technique}

The Fluorescence decay measurements of DDP dye with urea derivatives were recorded using IBH time correlated single-photon counting spectrometer with microchannel plate photomultiplier tube (MCP-PMT) (Hamamatsu, R3809U) as detector. A diode pumped millennia V CW laser (spectra physics) was used to pump the Ti-sapphire rod in a Tsunami picosecond mode-locked laser system (Spectra Physics). The $750 \mathrm{~nm}(85 \mathrm{MHz}$ ) beam from the Tisapphire laser was passed through a pulse picker (Spectra Physics, GWU 23PS) to generate $4 \mathrm{MHz}$ pulses. The second harmonic output was generated by a flexible harmonic generator (Spectra Physics, GWU 23PS). A vertically polarized $377 \mathrm{~nm}$ laser was used to excite the sample. The fluorescence of DDP dye was monitored at magic angle $\left(54.7^{\circ}\right)$. This was counted by a MCP-PMT apparatus (Hamamatsu R3809U) after being passed through the monochromatic and was preceded through a constant fraction discriminator (CFD), a time-to-amplitude converter (TAC) and a multichannel analyzer (MCA). The instrument response function for this system is $\sim 50 \mathrm{ps}$. The obtained fluorescence decays were analyzed by using IBH software (DAS-6) which is based on reconvolution technique using iterative nonlinear least squares method.

\section{Electrochemical impedance spectroscopy (EIS)}

EIS studies were recorded using $\mathrm{CH}$ INSTRUMENT CH1604E electrochemical analyzer. EIS study is also known as alternate current (AC) conductivity study. A plot of z' vs z" (Nyquist or colecole plots) taken over a wide frequency range $(50 \mathrm{~Hz}$ to $5 \mathrm{MHz}$ ) at room temperature was carried out.

\section{RESULTS AND DISCUSSION}

\section{Absorption spectral studies}

DDP dye exhibits two characteristic absorption peaks at 248 and $348 \mathrm{~nm}$ accompanied with a shoulder around $360 \mathrm{~nm}$ as reported [42]. Addition of BSA results no significant change in the absorbance at the longest wavelength absorption maximum of DDP dye (figure 3).The absorbance at the ICT absorption maximum remains unaltered even in the presence of very high concentration of BSA. The increase in the absorbance around $280 \pm 10 \mathrm{~nm}$ is attributed to the strong absorbance of BSA. Further, an isosbestic point in the absorption spectrum of DDP dye with BSA (figure 3 ) is correlated to the formation of a ground state complex. Our earlier studies of DDP dye with hydrogen-bonding assemblies of amides and urea derivatives also resulted in an isosbestic point which signifies the presence of equilibrium between dye and BSA. From absorption spectral studies it is evident that BSA associates with dye molecules in the aqueous phase. In the present investigation, we account for the role of BSA that possesses hydrogen-bonding as well as hydrophobic moieties on the variation in the excited state nature of DDP dye.

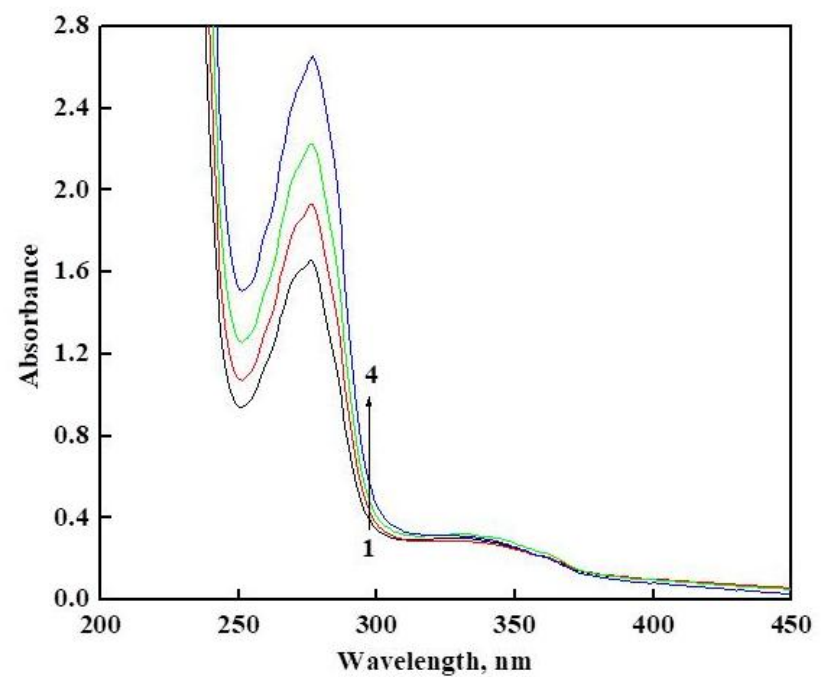

Fig-3: Absorption spectrum of DDP dye with BSA in water. 1. DDP dye $\left(7.14 \times 10^{-6} M\right)$. 2. DDP dye $\left(7.1 \times 10^{-6} M\right)+$ BSA $0.75 \times 10^{-5} \mathrm{M}$. 3. DDP dye $\left(7.1 \times 10^{-6} \mathrm{M}\right)+$ BSA $1.5 \times$ $10^{-5}$ M. 4. DDP dye $\left(7.1 \times 10^{-6} \mathrm{M}\right)+$ BSA $3.0 \times 10^{-5} \mathrm{M}$. 


\section{Emission spectral studies}

Addition of BSA to DDP dye results in a fluorescence enhancement (figure 4). A broad emission maximum around 430 to $440 \mathrm{~nm}$ resulted on the addition of BSA as observed in the case of urea, and a close observation on the emission spectra reveals the presence of two emission peaks of almost similar intensity separated by a few nm only. Interestingly, with a further increase in the concentration of BSA resulted in broad emission maxima at $435 \pm \mathrm{nm}$ with a larger fold of enhancement at the longest wavelength emission. The emission spectral studies of BSA with DDP dye reveals that the two emission peaks of DDP dye are partially separated and coalesce to form a single peak at higher concentration of BSA. The extent of fluorescence enhancement is accompanied with no considerable shift in the emission towards the red or blue region (figure 5).

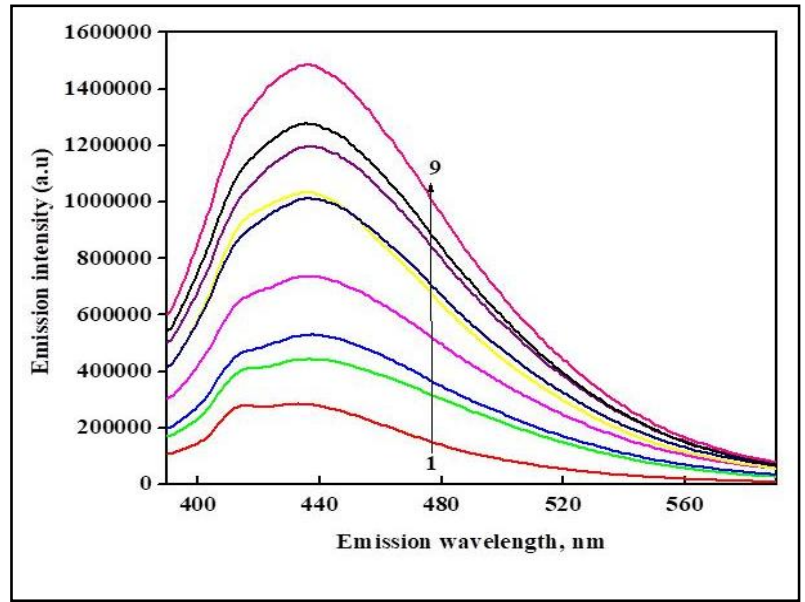

Fig-4: Emission spectra of DDP dye in the absence and presence of BSA in water. $\left.\lambda_{\mathrm{ex}} 360 \mathrm{~nm} .1\right)$ DDP dye alone $(7.1$ $\left.\left.\times 10^{-6} \mathrm{M}\right), 2\right)$ DDP dye $\left(7.1 \times 10^{-6} \mathrm{M}\right)+$ BSA $0.75 \times 10^{-5}$ $\mathrm{M}, 3)$ DDP dye $\left(7.1 \times 10^{-6} \mathrm{M}\right)+$ BSA $\left.1.5 \times 10^{-5} \mathrm{M}, 4\right)$ DDP dye $\left(7.1 \times 10^{-6} \mathrm{M}\right)+$ BSA $\left.3.0 \times 10^{-5} \mathrm{M}, 5\right) \mathrm{DDP}$ dye $\left(7.1 \times 10^{-6} \mathrm{M}\right)+$ BSA $\left.4.5 \times 10^{-5} \mathrm{M}, 6\right)$ DDP dye $\left(7.1 \times 10^{-}\right.$ $\left.{ }^{6} \mathrm{M}\right)+$ BSA $\left.6.0 \times 10^{-5} \mathrm{M}, 7\right)$ DDP dye $\left(7.1 \times 10^{-6} \mathrm{M}\right)+$ BSA 7.5 $\left.\times 10^{-5} \mathrm{M}, 8\right)$ DDP dye $\left(7.1 \times 10^{-6} \mathrm{M}\right)+$ BSA 9.0 $\times$ $\left.10^{-5} \mathrm{M}, 9\right)$ DDP dye $\left(7.1 \times 10^{-6} \mathrm{M}\right)+$ BSA $12.0 \times 10^{-5} \mathrm{M}$.

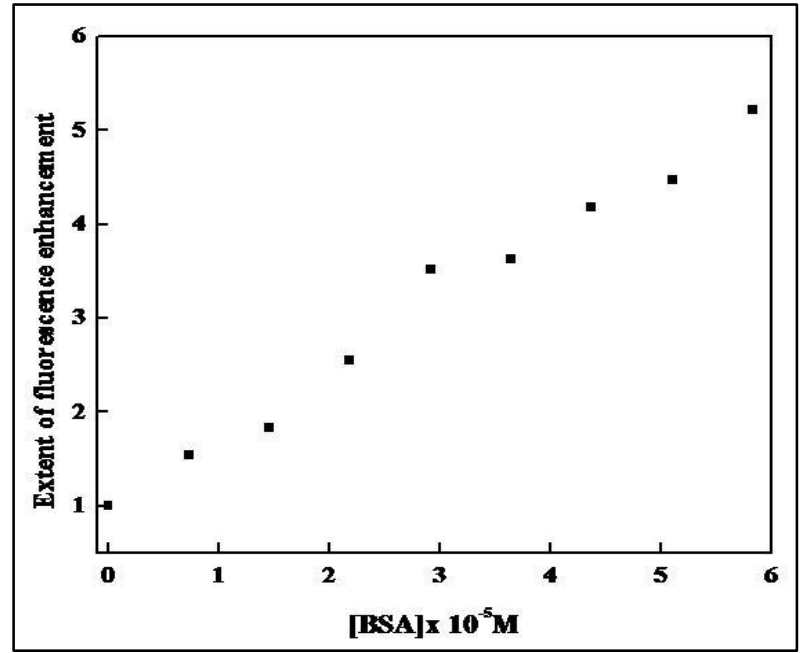

Fig-5: Extent of fluorescence enhancement of DDP dye with BSA
In general the excited state properties of intrinsic or extrinsic fluorophores are influenced by $\mathrm{pH}$, viscosity, dielectric constant, dipole moment and refractive index of the medium. The shift in the emission maximum towards the red or blue region is generally attributed to increase in the dipole moment of the medium or through hydrogen-bonding interaction or change in the polarity of the medium. In our present study there is no significant shift in the emission maxima of the dye upon the addition of BSA. Interaction of PET based acridinedione dyes with BSA resulted in a larger extent of fluorescence enhancement and shift in the emission maximum towards the blue region. The increase in the fluorescence intensity is attributed to the suppression of the PET process through space and the blue shift is attributed to the orientation of the dye predominantly in the hydrophobic pocket of BSA. BSA is a complex macromolecule which comprises several amino acids that contain polar and non-polar groups which induces hydrogen-bonding, hydrophobic interactions and electrostatic contributions. In our study the absence of any characteristic shift towards neither red nor blue region reveals that the dye is not oriented completely in the hydrophobic interior or hydrophilic exterior of BSA. The exact distribution of the dye could not be quantified from steady state measurements; rather DDP dye is distributed uniformly in an environment which is heterogeneous. This was established and elucidated based on the fluorescence lifetime decay studies only, which is discussed in detail in section 3.4.

It is well known that BSA binds with drugs and fluorescent probes and the binding constant values are calculated from the fluorescence intensity measurements, which signifies the extent of probe-protein binding. The binding constant of BSA with fluorescent probes are determined using the modified form of the BenesiHildebrand equation [47] which is so far the ideal tool in determining the nature of the interaction involving proteins $[46,32,33]$. Studies based on the interaction of probe/drug with BSA elucidate the importance of binding sites in BSA [18, 26, 32, 47-49]. The most preferred site for the binding of fluorescent probes in BSA is the hydrophobic exterior rather than the hydrophilic domain. The most probable location of the dye in BSA is ascertained by the binding constant value in comparison with other fluorescent probes. The binding site further provides valuable insight and information on the nature of the interaction and the stability of DDP dye with other ICT based dyes with BSA. Interaction of BSA with norharmane [22], AODIQ [29, 30], p-(dimethylaminobenzamidothiosemicarbazone)[43], methylene blue [54] and nile blue [53], reveals a higher binding constant. The binding constant value, which is of the order of $10^{4} \mathrm{M}^{-1}$ reveals that the dye is located in the hydrophobic interior of the protein molecule. We have employed the modified Benesi-Hildebrand equation interaction of DDP with BSA as applied to other probe-protein systems $[26,33,43]$.The binding constant of DDP dye with BSA 
is obtained from the plot of $\left(\mathrm{F}_{\mathrm{N}}-\mathrm{F}_{0}\right) /\left(\mathrm{F}_{\mathrm{x}}-\mathrm{F}_{0}\right)$ versus $1 /[\mathrm{BSA}]$. The binding constant $(\mathrm{K})$ calculated from the slope and the intercept is found to be $5.070 \times 10^{-3} \mathrm{M}^{-1}$ for DDP dye which is found to be similar to that of the ICT based probes interaction with BSA as proposed earlier. The binding constant value of DDP dye with BSA confirms that the dye-protein interaction is comparatively hydrophobic in nature. This is further supported by time-resolved fluorescence decay measurements. The literature reports portray that the interaction of drugs [47, 49] with globular proteins also results in high binding constant value which is correlated to the binding of the drug in the hydrophobic interior of protein molecule.

If hydrophobic influences on the excited state properties of DDP dye would have been more predominant than hydrogen-bonding influences, a blue shift in the emission maxima would have resulted. On the contrary, no blue shift in the emission results. The pattern of shift clearly reveals that apart from the hydrophilic and hydrophobic nature of BSA influence the emission spectra of DDP dye wherein the presence of microhetrogeneous population of the amino acids definitely governs the emissive nature of the dye. The fluorescence enhancement of DDP dye in the presence of BSA is larger in terms of fluorescence enhancement. The influence of urea-solvent hydrogenbonding properties on the photophysical behavior of fluorescent probe results either in an increase or decrease in the fluorescence emission and lifetime. This provides an excellent approach to study in depth regarding the interaction of water soluble fluorophores in the presence of large macromolecules.

\section{Mechanism of fluorescence enhancement of DDP dye with BSA}

In general, the mechanism of fluorescence enhancement (FE) of fluorophore in the presence of BSA in aqueous or buffered solutions is attributed to two different mechanisms [26, 51-54]. These mechanisms are postulated in regard to fluorescence enhancement of extrinsic probes involving globular proteins such that addition of BSA results in change in the absorption and emission spectral properties. A change in the microenvironment resulted by change in $\mathrm{pH}$ and viscosity by the addition of BSA influences the medium. One mechanism signifies that $\mathrm{FE}$ is due to the variation in the bulk viscosity and the polarity around the probe molecule. It was observed that the addition of protein result in the either decrease or increase in the polarity of the medium around the vicinity of the dye [57]. This mechanism signifies that the increase in the fluorescence intensity accompanied with a shift in the emission is attributed to the change in the microenvironment around the fluorophore resulting in the stabilization or destabilization of the charge transfer (CT) state.
Interaction of BSA and HSA with fluorescent probes involving a change in the polarity around the fluorophore has been well documented in the literature involving probe-protein interaction [32, 33]. Herein, DDP is an ICT based dye which exhibits only fluorescence enhancement and no characteristic shift is observed. On the contrary, another mechanism signifies an intermolecular energy transfer from the protein molecule to the probe resulting in a FE [55-58]. This mechanism was ruled out since there were no new emissive peaks arising from DDP dye on the addition of BSA. The emission intensity in the spectral range of 435 \pm 10 was almost similar which authenticates that the local excited (LE) state emission is stabilized to a larger extent. This was confirmed from 3D emission contour spectral studies.

Apart from these mechanisms, FE is also correlated to the binding of the dye to the protein molecule resulting in the formation of a stable complex in the excited state [48] such that free dye and bound dye exists in solution. This mechanism was ruled out in our present study since there exist no free dye component in aqueous phase on the immediate addition of BSA. This was established based on the fluorescence lifetime amplitude distribution of the various lifetime components of DDP dye.

Interestingly, the addition of urea (hydrophilic and hydrophobic moieties) results in a complete change in the microenvironment. The increase in the fluorescence intensity of DDP dye on the addition of urea results in a red shift in the emission maximum and this is attributed to hydrogen-bonding interaction. This mechanism results in the stabilization or destabilization of the CT or LE state emission of the flourophore. The presence of urea around the dye molecules compete with water molecules in forming hydrogen-bonding interaction such that the variation in the microenvironment of dye is influenced by the concentration of urea. The nature of the solute influences the excited state properties of DDP dye is illustrated from this present study.

\section{Emission Contour spectral studies}

The 3D fluorescence spectrum of DDP dye exhibits a single contour of emission intensity in the spectral range of $435 \pm 10 \mathrm{~nm}$ that corresponds to 370 $\pm 5 \mathrm{~nm}$ (excitation wavelength) in the absence of BSA as shown in figure $6 \mathrm{a}$. The pattern of contour is uniform and the concentric circles around the emission maximum are almost uniform and no appreciable variation in the intensity results. The excitation wavelength scan interval was varied from 280 to 400 $\mathrm{nm}$ and the emission wavelength scan interval was from 340 to $500 \mathrm{~nm}$. 3D contour spectra of DDP dye on the addition of BSA results in a complete change in the pattern and shape of the contour (figure $6 \mathrm{~b}$ ). The emission intensity at the maxima is much broader and there exists no uniform pattern of concentric circles 
which clearly reveal that the excited state characteristics of DDP dye are completely governed by the introduction of protein molecule. The contour spectra also illustrates that no new emission results from the dye in the presence of BSA which also supports our proposed mechanism of fluorescence enhancement.

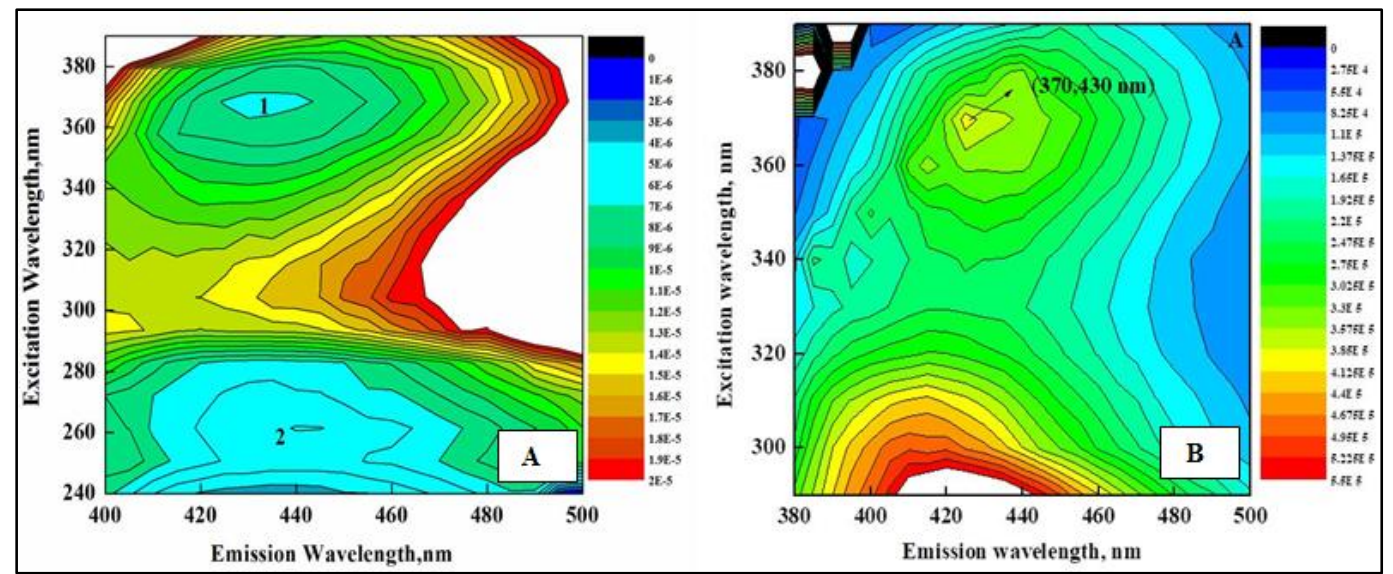

Fig-6: 3D Emission contour spectral studies of Dye with various concentrations of BSA. (A) Dye alone, (B) Dye with BSA

\section{Time Resolved Fluorescence Studies}

The fluorescence life time of DDP dye exhibits tri-exponential decay in water. The existence of multi exponential behavior is attributed to the orientation of the acceptor moieties in plane with the pyran ring and perpendicular to the pyran ring structure such that two different conformations can coexist with certain difference in their conformational stability and relative proportion of existence in the presence of water molecules [42]. Of the three lifetime components, there exists one major lifetime component of $6.81 \mathrm{~ns}$. Addition of BSA results in a marked change in the fluorescence lifetimes of all the components with significant variation in the relative amplitude distribution. The fluorescence decay of DDP dye with BSA is shown in figure 7 and the lifetime values are provided in table 1 .

BSA, which contains three domains, has two binding sites namely site I and site II. The binding affinity offered by site (I) is mainly through hydrophobic interaction, whereas the site (II) involves the combination of hydrophobic, hydrogen-bonding, hydrophilic and electrostatic interactions [50,51]. Timeresolved fluorescence decay measurements illustrate that the DDP dye is largely confined to the binding site (I) of BSA, which is predominantly influenced by the hydrophobic interactions. From the fluorescence lifetime decay analysis of DDP dye in the presence of highest BSA concentration, the shorter component $(0.4 \mathrm{~ns})$ is assigned to dye prominently located in the aqueous phase with lesser number of protein molecule surrounding the close vicinity of dye. The other two lifetimes are attributed to the dye molecule located in the hydrophilic phase $(2.0 \mathrm{~ns})$ of the protein molecule and the dye molecule deeply buried in the hydrophobic interior $(7.3 \mathrm{~ns})$ of the BSA. A trimodal behaviour illustrates that DDP dye is located in multi heterogeneous environment created by the protein molecule. The fluorescence lifetime of the free unbound dye component (surrounded by water molecules only) is also influenced by the presence of several amino acid molecules, whereas the relative amplitude decreases with increasing concentration of BSA.

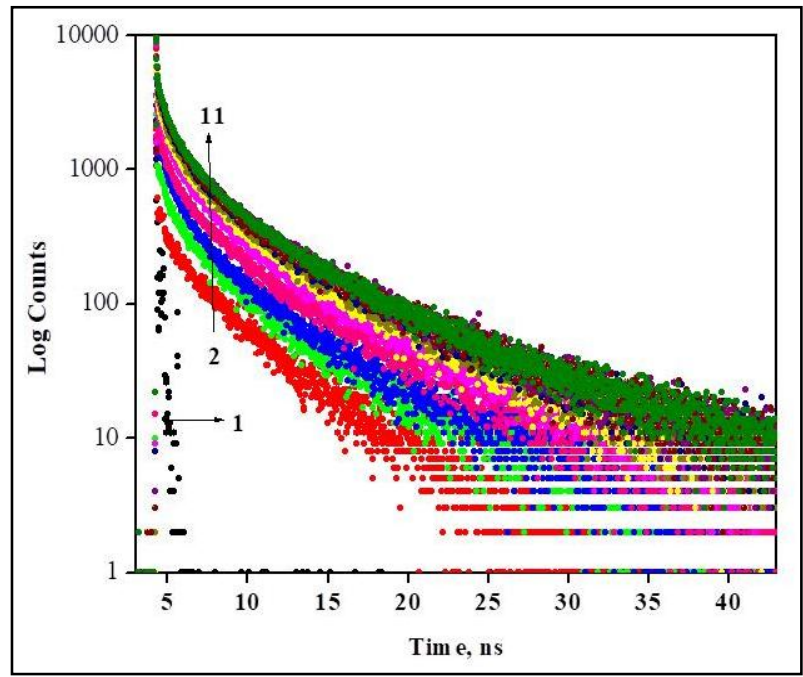

Fig-7: Fluorescence lifetime decay of DCM dye with BSA in water. $\left.\lambda_{\mathrm{ex}} 360 \mathrm{~nm} .1\right)$ Laser profile, 2) DDP dye alone $\left(7.1 \times 10^{-6} \mathrm{M}\right.$ ) , 3) DDP dye $\left(7.1 \times 10^{-6} \mathrm{M}\right)+$ BSA $\left.0.75 \times 10^{-5} \mathrm{M}, 4\right) \mathrm{DDP}$ dye $\left(7.1 \times 10^{-6} \mathrm{M}\right)+$ BSA $\left.1.5 \times 10^{-5} \mathrm{M}, 5\right)$ DDP dye $\left(7.1 \times 10^{-6} \mathrm{M}\right)$ + BSA 3.0 $\left.\times 10^{-5} \mathrm{M}, 6\right)$ DDP dye $\left(7.1 \times 10^{-6} \mathrm{M}\right)+$ BSA $4.5 \times 10^{-5}$ M, 7) V DDP dye $\left(7.1 \times 10^{-6} \mathrm{M}\right)+$ BSA $\left.6.0 \times 10^{-5} \mathrm{M}, 8\right)$ DDP dye $\left(7.1 \times 10^{-6} \mathrm{M}\right)+$ BSA $\left.7.5 \times 10^{-5} \mathrm{M}, 9\right) \mathrm{DDP} \operatorname{dye}\left(7.1 \times 10^{-6} \mathrm{M}\right)+$ BSA 9.0 $\left.\times 10^{-5} \mathrm{M}, 10\right)$ DDP dye $\left(7.1 \times 10^{-6} \mathrm{M}\right)+$ BSA $10.5 \times 10^{-5}$ M, 11) DDP dye $\left(7.1 \times 10^{-6} \mathrm{M}\right)+$ BSA BSA $12.0 \times 10^{-5} \mathrm{M}$.

Time resolved fluorescence spectral technique is used as an efficient tool in resolving the most probable location of the fluorophore in different environments. From the above observation it is clear that the concentration of BSA play a significant role in the fluorescence lifetime of DDP dye in aqueous solution that are governed by hydrophobic influences accompanied with different hydrogen-bonding moieties. 
Table-1: Fluorescence lifetime decay analysis of DCM dye with BSA in water

\begin{tabular}{|llllllll}
\hline BSA & $\mathrm{x}$ & & & & & \\
& & & & & & \\
$10^{-5}(\mathrm{M})$ & $\tau_{1}(\mathrm{~ns})$ & $\tau_{2}(\mathrm{~ns})$ & $\tau_{3}(\mathrm{~ns})$ & $\mathrm{B}_{1}(\%)$ & $\mathrm{B}_{2}(\%)$ & $\mathrm{B}_{3}(\%)$ & $\chi^{2}$ \\
\hline 0.0 & 0.18 & 3.51 & - & 49 & 57 & - & 1.80 \\
\hline 0.75 & 0.61 & 4.00 & - & 32 & 68 & - & 1.21 \\
\hline 1.5 & 0.85 & 4.78 & - & 43 & 56 & - & 1.21 \\
\hline 3.0 & 0.34 & 1.9 & 6.6 & 13 & 35 & 52 & 1.02 \\
\hline 4.5 & 0.31 & 2.76 & 7.1 & 13 & 38 & 49 & 1.04 \\
\hline 6.0 & 0.48 & 2.37 & 7.95 & 23 & 28 & 39 & 1.09 \\
\hline 7.5 & 0.50 & 2.32 & 7.72 & 22 & 37 & 41 & 1.06 \\
\hline 9.0 & 0.49 & 2.25 & 7.47 & 21 & 36 & 43 & 1.08 \\
\hline 10.5 & 0.48 & 2.24 & 8.04 & 22 & 38 & 40 & 1.09 \\
\hline 12.0 & 0.39 & 2.01 & 7.29 & 13 & 37 & 50 & 1.05 \\
\hline
\end{tabular}

Electrochemical Impendence spectroscopy (EIS)

A plot of z vs z' (Nyquist or cole-cole plots) taken over a wide frequency range $(50 \mathrm{~Hz}$ to $5 \mathrm{MHz})$ at room temperature and fitted with equivalent circuit as shown in supporting information figure 8. An equivalent circuit of charge transfer capacitance $\left(\mathrm{C}_{\mathrm{ct}}\right)$ and charge transfer resistance, $\left(\mathrm{R}_{\mathrm{ct}}\right)$ was in parallel connection with resistance in series $\left(R_{s}\right)$. The number of environment involved in the charge transfer process is equal to the number of equivalent circuits. The capacitive behavior of free dye with three equivalent circuits representing three environments as, i) a semicircle is observed at high frequencies, attesting the charge transfer at the electrode / solution interface, ii) a transition zone at intermediary frequencies, iii) a Warburg like line at low frequencies characteristic for diffusion process. The $\mathrm{R}(\mathrm{CR})$ depicts the Randels circuit of an electrochemical cell which ensures the electrostatic mode of attraction between the dye and BSA molecule. On addition of BSA, to DDP dye we observe that a charge transfer capacitance $C_{c t}$ and charge transfer resistance, $\mathrm{R}_{\mathrm{ct}}$ decrease and this has been confirmed with equivalent circuits.

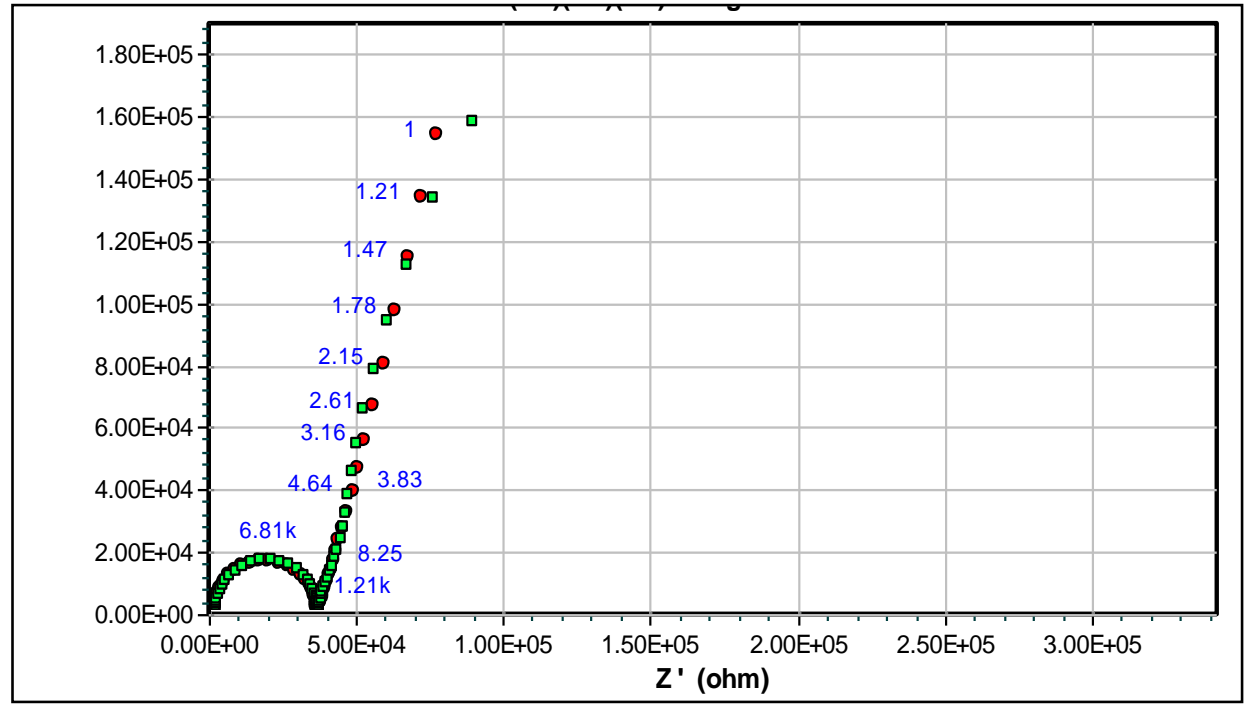

Fig-8: Electrochemical Impedance Spectroscopy of DDP dye in water in the presence of BSA.

\section{CONCLUSION}

DDP dye interaction with a globular protein by various techniques like UV-Visible, fluorescence spectroscopy and Electrochemical Impedance Studies (EIS) were employed as a tool in establishing the most probable orientation of the dye in a micro heterogeneous environment. These techniques provide link in establishing the binding nature as well as preferred mode of interaction of DDP dye with BSA. The fluorescence enhancement reveals that the excited state properties are largely governed by the concentration of the protein. Presence of micro heterogeneous environment in aqueous medium is confirmed by the triexpontential decay lifetime of DDP dye and a similar inference is observed in EIS by the Warburg formation of more than one layer.
Spectroscopic studies reveal that electrochemistry coupling with fluorescence spectral techniques certainly provide an efficient way to characterize both the binding mode of DDP dye with water soluble biomolecules.

\section{ACKNOWLEDGEMENT}

The authors are thankful to Prof. P. Ramamurthy and Dr. C.Selvaraju of National Centre for Ultrafast Processes, University of Madras, Chennai-600 113, India for permitting us to avail the fluorescence emission and contour facilities. The authors are thankful for the support and facilities for carrying out spectral studies provided by the Secretary, Shri Ashok Kumar Mundra and Principal, Dr. R.Ganesan, Dwaraka Doss Goverdhan Doss Vaishnav College,Arumbakkam, 
Chennai-106. R.K thank Dr S.Ilangovan, Former Head of the Department of Chemistry Dwaraka Doss Goverdhan Doss Vaishnav College, Chennai 600106 for his constant support and encouragement.

\section{REFERENCES}

1. Kragh-Hansen, U. (1981). Molecular aspects of Ligand binding to serum albumin, Pharmacol. Rev. 33,17-53.

2. Lakowicz, J.R. (1996). Principles of Fluorescence Spectroscopy, Kluwer Academic Plenum Publications, $3^{\text {rd }}$ ed, $12,1166$.

3. Huang, B.X., Kim, H.Y., Dass, C. (2000). Probing three-dimensional structure of Bovine Serum Albumin by chemical cross-linking and Mass Spectrometry, J. Am. Soc. Mass. Spectrom, 15, 1237-1247.

4. Curry, S., Mandelkow, H., Brick, P., Franks, N. (1998). Crystal structure of human Serum Albumin complexed with fatty Acids reveals an Asymmetric distribution of Binding sites, Nat. Sci. Biol, 5, 827835.

5. Carter, D.C., Chang, B.; Ho, J.X., Keeling, K., Krishnasami, Z. (1994). Preliminary Crystallographic studies of four Crystal forms of Serum Albumin, Eur. J. Biochem. 226,1049-1052.

6. Brown, J.R., Shockley, P. (1982). In "Lipid-Protein Intractions", P.Jost and O.Griffith, ( eds 1),New York, John Wiley \& Sons, 1,25.

7. Hartley, R. W., Peterson, E. A., Sober, H. A. (1962).The relation of free Sulfhydryl groups to Chromotographic Hetergeneity Biochemistry, 1,60.

8. Peterson, E.A., Foster, J.F. (1965).The Micro Heterogeneity of Plasma Albumins, J. Biol. Chem, 240,2503-2507.

9. Figgie, J., Rossing, T. H., Fencl, V. (1991). The Role of Serum Proteins in Acid-Base Equilibria, J . Lab. Clin. Med. 117,453-467.

10. Carter, D.C., Ho, J.X. (1994). Structure of Serum Albumin, Adv. Prot. Chem, 45,153-203.

11. Iqbal, M.,Veroll, R.E. (1987).Volumetric properties of Aqueous solution of Bovine Serum Albumin, Human Serum Albumin and Human Hemoglobin, J. Phys. Chem. 91,1935-1941.

12. He, X.M., Carter, D.C. (1992). Atomic Structure and Chemistry of Human Serum Albumin, Nature, 358,209-215.

13. Dockal, M., Carter, D.C., Ruker, F. (1999). The Three recombinant domains of Human Serum Albumin, J. Biol. Chem. 274,29303-29310.

14. Kauzmann, W. (1959). Some Factors in the Interpretation of Protein Denaturation, Adv. Prot. Chem, 14,1-63.

15. Weder, H. J. (1970). Experimental methods, Teatment of Experimental data and Thermodynamics of Binding Reactions of Thymoleptic Drugs and model dyes, J. Pharm. Sci 59,1563-1569.

16. Pal, M. K, Patra. S.K.(1994). Fluorimetric probes of Individual and Competitive Binding of 1-
AnilinoNapthalene-8-Sulfonate,eosine and fluorescene to Bovine Serum Albumin, J Biochem Biophys, 31,109-114.

17. Waheed, A., Rao. K.S., Gupta, P.D. (2000). Mechanism of Dye Binding in the Protein Assay using eosin Dyes,Anal. Biochem, 287,73-79.

18. Nag.A,Kankan Bhattacharyya. (1990). Role of Twisted Intramolecular Charge Transfer in the Fluorescence Sensitivity of Biological Probes Diethylaminocoumarin Laser Dyes, Chem. Phys. lett. 169,12-16.

19. Hrishfield, K. M,Dimitri Toptygin,Gopal Grandhige. (1996),Steady-State and TimeResolved Fluorescence Measurements for studying Molecular Interaction;Interaction of a CalciumBinding Probe with Proteins, Biophy. Chem. 622,25-38.

20. Nerli, B., Pico, G. (1994). Influence of the medium conditions on the 1-Anilino-8-Napthalene Sulfonate Bovine Serum Binding, Physicol. Biochem. Biophys, 102,5-8.

21. Avdulov, N.A., Wood, W.G. (1996). Direct Binding of Ethanol to Bovine Serum Albumin: A Fluorescent and 13C-NMR multiplet Relaxation Study, Biochem, 35,340-347.

22. Mallick, A., \& Chattopadhyay, N. (2005). Photophysics in Motionally constrained Bioenvironment: Interactions of Norharmane with Bovine Serum AlbuminII. Photochemistry and photobiology, 81(2), 419-424.

23. Guharay, J., Sengupta, B., \& Sengupta, P. K. (2001). Protein-flavonol interaction: fluorescence spectroscopic study. Proteins: Structure, Function, and Bioinformatics, 43(2), 75-81.

24. Takeda, K., Yoshida, I., \& Yamamoto, K. (1991). Changes of fluorescence lifetime and rotational correlation time of bovine serum albumin labeled with 1-dimethylaminonaphthalene-5-sulfonyl chloride in guanidine and thermal denaturations. Journal of protein chemistry, 10(1), 17-23.

25. Weber, G., \& Farris, F. J. (1979). Synthesis and spectral properties of a hydrophobic fluorescent probe: 6-propionyl-2-(dimethylamino) naphthalene. Biochemistry, 18(14), 3075-3078.

26. Lunardi, C. N., Tedesco, A. C., Kurth, T. L., \& Brinn, I. M. (2003). The complex between 9-(ndecanyl) acridone and Bovine Serum Albumin. Part 2. What do fluorescence probes probe?. Photochemical \& Photobiological Sciences, 2(9), 954-959.

27. Wilton, D. C. (1990). The fatty acid analogue 11(dansylamino) undecanoic acid is a fluorescent probe for the bilirubin-binding sites of albumin and not for the high-affinity fatty acid-binding sites. Biochemical Journal, 270(1), 163-166.

28. Das, R., Mitra, S., Nath, D., \& Mukherjee, S. (1996). Excited state proton transfer reaction as a probe for the microenvironment of a binding site of 
bovine serum albumin: effect of urea. The Journal of Physical Chemistry, 100(34), 14514-14519.

29. Mallick, A., \& Chattopadhyay, N. (2005). Photophysics in Motionally constrained Bioenvironment: Interactions of Norharmane with Bovine Serum AlbuminףI. Photochemistry and photobiology, 81(2), 419-424.

30. Mallick, A., Haldar, B., \& Chattopadhyay, N. (2005). Spectroscopic investigation on the interaction of ICT probe 3-acetyl-4-oxo-6, 7dihydro-12H indolo-[2, 3-a] quinolizine with serum albumins. The Journal of Physical Chemistry B, 109(30), 14683-14690.

31. Bultot, L., Horman, S., Neumann, D., Walsh, M. P., Hue, L., \& Rider, M. H. (2009). Myosin light chains are not a physiological substrate of AMPK in the control of cell structure changes. FEBS letters, 583(1), 25-28.

32. Jisha, V. S., Arun, K. T., Hariharan, M., \& Ramaiah, D. (2006). Site-selective binding and dual mode recognition of serum albumin by a squaraine dye. Journal of the American Chemical Society, 128(18), 6024-6025.

33. Kalyanasundaram, K. (2012). Photochemistry in microheterogeneous systems. Elsevier.

34. Woods, L. L. (1958). Some further reactions of 2 , 6-dimethyl-4-pyrone. Journal of the American Chemical Society, 80(6), 1440-1442.

35. Meyer, M., \& Mialocq, J. C. (1987). Ground state and singlet excited state of laser dye DCM: Dipole moments and solvent induced spectral shifts. Optics communications, 64(3), 264-268.

36. Hammond, P. R. (1979). Laser dye DCM, its spectral properties, synthesis and comparison with other dyes in the red.Optics Communications, 29(3), 331-333.

37. Hsing-Kang, Z., Ren-Lan, M., Er-pin, N., \& Chu, G. (1985). Behaviour of the laser dye 4dicyanomethylene-2-methyl-6-

dimethylaminostryryl-4H-pyran in the excited singlet state. Journal of photochemistry, 29(3-4), 397-404.

38. Taylor, J. R. (1986). Passive mode locking of DCM and Rhodamine 101 flashlamp-pumped dye laser systems. Optics communications, 57(2), 117-120.

39. Bourson, J., Doizi, D., Lambert, D., Sacaze, T., \& Valeur, B. (1989). A derivative of laser dye DCM highly soluble in alcohols. Optics communications, 72(6), 367-370.

40. Marason, E. G. (1981). Laser dye DCM: CW, synchronously pumped, cavity pumped and singlefrequency Communications, 37(1), 56-58. performance. Optics

41. Antonov, V. S., \& Hohla, K. L. (1983). Dye stability under excimer-laser pumping. Applied Physics B, 30(3), 109-116.

42. Gayathri, S., Vasanthi, R., Vanjinathan, M., \& Kumaran, R. (2018). Photophysical and Electrochemical studies of 4-dicyanomethylene 2,
6-dimethyl-4H-pyran (DDP) dye with amides in water. Journal of fluorescence, 28(6), 1379-1391.

43. Gayathri, S., Vasanthi, R., Vanjinathan, R. M., \& Kumaran, R. (2019). Spectroelectrochemical Investigation of 4-Dicyanomethylene 2, 6Dimethyl-4H-Pyran (DDP) Dye with Guanidine Hydrochloride $(\mathrm{GuHCl})$ in Water. J. Mater. Sci. Res, 8, 6-17.

44. Somasundaram, G., Rajaraman, V., Mahalingam, V., Dhenadhayalan, N., \& Rajendran, K. (2020). Fluorescence coupled with electrochemical approach at the bulk and the interface region of hydrogen-bonding self assemblies of urea derivatives with DDP dye in aqueous solution. Spectrochimica Acta Part A: Molecular and Biomolecular Spectroscopy, 229, 117914.

45. Somasundaram, G., Rajaraman, V., Mahalingam, V., \& Rajendran, K. (2019). Electrostatic Investigation of 4-Dicyanomethylene-2, 6dimethyl-4 H-pyran (DDP) Dye with Amide Derivatives in Water. ACS Omega.

46. Sowntharya, C., Gayathri, S., Dhenadayalan, N., Vasanthi, R., Vanjinathan, M., \& Kumaran, R. (2017). Photophysical studies of a food hydrocolloid, Gum Arabic with resorcinol based acridinedione dyes in water.Journal of Photochemistry and Photobiology A: Chemistry, 341, 78-86.

47. Benesi, H. A., \& Hildebrand, J. H. J. (1949). A spectrophotometric investigation of the interaction of iodine with aromatic hydrocarbons. Journal of the American Chemical Society, 71(8), 2703-2707.

48. Wu, F. Y., Ji, Z. J., Wu, Y. M., \& Wan, X. F. (2006). Interaction of ICT receptor with serum albumins in aqueous buffer. Chemical physics letters, 424(4-6), 387-393.

49. Hu, Y. J., Liu, Y., Zhao, R. M., Dong, J. X., \& Qu, S. S. (2006). Spectroscopic studies on the interaction between methylene blue and bovine serum albumin. Journal of Photochemistry and Photobiology A: Chemistry, 179(3), 324-329.

50. Zhao, H., Ge, M., Zhang, Z., Wang, W., \& Wu, G. (2006). Spectroscopic studies on the interaction between riboflavin and albumins. Spectrochimica Acta Part A: Molecular and Biomolecular Spectroscopy, 65(3-4), 811-817.

51. Li, Y., He, W., Dong, Y., Sheng, F., \& Hu, Z. (2006). Human serum albumin interaction with formononetin studied using fluorescence anisotropy, FT-IR spectroscopy, and molecular modeling methods. Bioorganic \& medicinal chemistry, 14(5), 1431-1436.

52. He, W., Li, Y., Si, H., Dong, Y., Sheng, F., Yao, X., \& Hu, Z. (2006). Molecular modeling and spectroscopic studies on the binding of guaiacol to human serum albumin. Journal of Photochemistry and Photobiology A: Chemistry, 182(2), 158-167.

53. Lee, S. H., Suh, J. K., \& Li, M. (2003). Determination of bovine serum albumin by its enhancement effect of Nile blue 
fluorescence. BULLETIN-KOREAN CHEMICAL SOCIETY, 24(1), 45-48.

54. Lakowicz, J. R. (1999). Principles of fluorescence spectroscopy Kluwer. New York.

55. Wang, F., Yang, J., Wu, X., Sun, C., Liu, S., Guo, C., \& Jia, Z. (2005). The interaction mechanism and fluorescence enhancement in morin-Al3+sodium dodecyl benzene sulfonate-protein system. Chemical physics letters, 409(1-3), 14-22.

56. Wang, F., Yang, J., Wu, X., Sun, C., Liu, S., Guo, C., \& Wang, F. (2005). Fluorescence enhancement effect for the determination of proteins with morinAl3+-cetyltrimethylammonium bromide. Talanta, 67(4), 836-842.
57. Wang, F., Yang, J., Wu, X., Wang, X., Sun, C., Liu, S., \& Guo, C. (2006). Molecular selfassembling and the fluorescence enhancement in morin-Al3+-cetyltrimethylammonium bromideprotein system. Biochimie, 88(2), 121-129.

58. Guo, C., Wu, X., Yang, J., Wang, F., Jia, Z., Ran, D., \& Zheng, J. (2006). Determination of proteins using fluorescence enhancement of $\mathrm{Tb} 3+$ benzoylacetone-sodium dodecyl benzene sulfonate-protein system. Journal of Photochemistry and Photobiology A: Chemistry, 181(1), 50-55. 